\title{
Third-order Douglas-Kroll ab initio model potential for actinide elements
}

\author{
Jozef Paulovič, Takahito Nakajima, and Kimihiko Hirao ${ }^{\text {a) }}$ \\ Department of Applied Chemistry, School of Engineering, The University of Tokyo, Tokyo 113-8656, Japan \\ Luis Seijo \\ Departamento de Química and Instituto Universitario de Ciencia de Materiales Nicolás Cabrera, C-XIV, \\ Universidad Autónoma de Madrid, E-28049 Madrid, Spain
}

(Received 12 February 2002; accepted 16 April 2002)

\begin{abstract}
A relativistic ab initio model potential (AIMP) method with the third-order Douglas-Kroll (DK3) approximation has been developed for the whole series of the actinide elements from Th to Lr. Two different cores, i.e., $[\mathrm{Xe}, 4 f, 5 d]$ and $[\mathrm{Xe}, 4 f]$, have been employed and the corresponding valence basis sets, $(14 s 10 p 11 d 9 f) /[6 s 5 p 5 d 4 f]$ and $(14 s 10 p 12 d 9 f) /[6 s 5 p 6 d 4 f]$, are presented for all actinides. The mean absolute errors of the AIMP relative to the all-electron results for the atomic SCF valence orbital energies $(\epsilon)$ and the radial expectation values $(\langle r\rangle)$ are $0.003(0.001)$ hartree and $0.004(0.006)$ bohr with the small (large) core set. The spectroscopic properties of the ${ }^{1} \Sigma^{+}$ ground state of thorium monoxide, ThO, are calculated at the SCF and complete active space SCF levels. The DK3-AIMP results again satisfactorily reproduce the all-electron DK3 results. The large core set gives almost the same results as the small set for atomic and molecular calculations, suggesting that the $5 d$ electrons can safely be omitted from the valence electrons in actinide chemistry. (C) 2002 American Institute of Physics. [DOI: 10.1063/1.1483850]
\end{abstract}

\section{INTRODUCTION}

The chemistry of $f$ elements, lanthanides, and actinides has received much attention. This is because of their fascinating complexity due to the possible open $f$ shells. They pose a great challenge to experimental and theoretical analysis. For actinides, both the electron correlation and relativistic effects have to be fully taken into account in order to get reliable results. The most widely used quantum mechanical method in the chemistry of lanthanides and actinides nowadays is the effective core potential (ECP) approximation. ${ }^{1-6}$ In this approximation the core electrons are modeled using a suitable function, and only the valence electrons are treated explicitly. Part of the relativistic effects, especially the scalar effects, may also be taken into account without having to perform full relativistic calculations.

In many cases it gives reasonable results while reducing computational effort. The $a b$ initio model potential (AIMP) method $^{7,8}$ has been developed as an extension of the model potential methods ${ }^{2-6}$ and describes the correct behavior for the inner nodal structure of the valence orbitals. The AIMP consists of a Coulomb potential, an exchange potential, and a projection operator, and has a clear physical meaning since it represents Coulomb and exchange interactions between a single valence electron and the core electrons. The implementation requires only modification of the one-electron operator in the usual AIMP methods.

Several sets of relativistic pseudopotentials are currently available in the literature. ${ }^{9-20}$ However, relativistic effects for the heavy elements such as actinides are very significant and a highly accurate treatment is required, even at the scalar relativistic level of theory. The main purpose of this study is

${ }^{a)}$ Electronic mail: hirao@qcl.t.u-tokyo.ac.jp to develop highly accurate AIMP for all actinide elements from Th to Lr by means of the third-order Douglas-Kroll (DK3) approximation. ${ }^{21,22}$ In order to check the performance of the present AIMP, illustrative calculations on the spectroscopic constants for the ${ }^{1} \Sigma^{+}$ground state of thorium monoxide, ThO, have been carried out using SCF and complete active space SCF (CASSCF) methods. The results are compared with the corresponding all-electron calculations.

\section{AB INITIO MODEL POTENTIAL AND VALENCE BASIS SET}

Relativistic effects were incorporated by the third-order Douglas-Kroll approximation. ${ }^{21,22}$ The no-pair DK3 Hamiltonian ${ }^{22}$ is given by

$$
H_{+}^{\mathrm{DK} 3}=H_{+}^{\mathrm{DK} 2}+\frac{1}{2} \sum_{i}\left[W_{1}(i),\left[W_{1}(i), V_{\mathrm{eff}}(i)\right]\right],
$$

where $H_{+}^{\mathrm{DK} 2}$ is the second-order Douglas-Kroll (DK2) Hamiltonian ${ }^{23-25}$ and can be written as

$$
\begin{aligned}
H_{+}^{\mathrm{DK} 2}= & \sum_{i} E_{i}-m c^{2}+\sum_{i} V_{\mathrm{eff}}(i)+\frac{1}{2} \sum_{i \neq j} V_{\mathrm{eff}}(i, j) \\
& -\frac{1}{2} \sum_{i}\left[W_{1}(i),\left[W_{1}(i), E_{i}\right]_{+}\right]_{+},
\end{aligned}
$$

with

$$
\begin{aligned}
& E_{i}=\left(p_{i}^{2} c^{2}+m^{2} c^{4}\right)^{1 / 2}, \\
& V_{\mathrm{eff}}(i)=A_{i}\left[V_{\mathrm{ext}}(i)+\left(\boldsymbol{\sigma}_{i} \mathbf{R}_{i}\right) V_{\mathrm{ext}}(i)\left(\boldsymbol{\sigma}_{i} \mathbf{R}_{i}\right)\right] A_{i},
\end{aligned}
$$




$$
\begin{aligned}
V_{\mathrm{eff}}(i, j)= & A_{i} A_{j}\left[\frac{1}{r_{i j}}+\left(\boldsymbol{\sigma}_{i} \mathbf{R}_{i}\right) \frac{1}{r_{i j}}\left(\boldsymbol{\sigma}_{i} \mathbf{R}_{i}\right)+\left(\boldsymbol{\sigma}_{j} \mathbf{R}_{j}\right) \frac{1}{r_{i j}}\left(\boldsymbol{\sigma}_{j} \mathbf{R}_{j}\right)\right. \\
& \left.+\left(\boldsymbol{\sigma}_{i} \mathbf{R}_{i}\right)\left(\boldsymbol{\sigma}_{j} \mathbf{R}_{j}\right) \frac{1}{r_{i j}}\left(\boldsymbol{\sigma}_{i} \mathbf{R}_{i}\right)\left(\boldsymbol{\sigma}_{j} \mathbf{R}_{j}\right)\right] A_{i} A_{j}, \\
A_{i}= & \left(\frac{E_{i}+m c^{2}}{2 E_{i}}\right)^{1 / 2}, \\
\mathbf{R}_{i}= & \frac{c \mathbf{p}_{i}}{E_{i}+m c^{2}}
\end{aligned}
$$

where $V_{\text {ext }}$ is the external potential, $m$ is the mass, $c$ is the speed of light, $\mathbf{p}_{i}$ is the momentum operator, and $\boldsymbol{\sigma}_{i}$ denotes the vector of three Pauli $2 \times 2$ spin matrices. Here, $W_{1}(i)$ is an integral operator with kernel,

$$
W_{1}\left(\mathbf{p}_{i}, \mathbf{p}_{i}^{\prime}\right)=A_{i}\left(\mathbf{R}_{i}-\mathbf{R}_{i}^{\prime}\right) A_{i}^{\prime} \frac{V_{\mathrm{ext}}\left(\mathbf{p}_{i}, \mathbf{p}_{i}^{\prime}\right)}{E_{i}+E_{i}^{\prime}},
$$

and $[a, b]_{+}$and $[a, b]$ denote the anticommutator and the commutator, respectively. For light elements, the DK2 approximation in Eq. (2) is sufficient. However, for heavy elements, with $Z$ larger than 80, the DK3 approximation is indispensable for an accurate description of relativistic effects.

In this study, the spin-free part of the DK3 Hamiltonian was used and the spin-dependent term was not considered. The relativistic kinematics correction to the two-electron integrals was ignored, since the effects of two-electron integrals have been shown to be small. Modification of the oneelectron integrals for the third-order relativistic correction with the DK3 Hamiltonian is not expensive in comparison with the DK2 Hamiltonian. The speed of light in a vacuum was taken to be 137.0359895 a.u.

The spin-free valence-only DK3-AIMP Hamiltonian with approximations described above is given as

$$
\begin{aligned}
H^{\mathrm{DK} 3-\mathrm{AIMP}}= & \sum_{i=1}^{N_{\nu}}\left(E_{i}-m c^{2}+V_{\mathrm{eff}}(i)\right. \\
& -\frac{1}{2}\left[W_{1}(i),\left[W_{1}(i), E_{i}\right]_{+}\right]_{+} \\
& +\frac{1}{2}\left[W_{1}(i),\left[W_{1}(i), V_{\mathrm{eff}}(i)\right]\right] \\
& +\sum_{\mu}\left[\frac{Z_{\mu}^{\mathrm{core}}}{\left|r_{i}-R_{\mu}\right|}+V_{\mu}^{\mathrm{DK} 3-\mathrm{AIMP}}\right] \\
& \left.+\sum_{i>j=1}^{N_{\nu}} \frac{1}{r_{i j}}+\sum_{\mu>\nu} \frac{Z_{\mu}^{\mathrm{eff}} Z_{\nu}^{\mathrm{eff}}}{\left|R_{\mu}-R_{\nu}\right|}\right),
\end{aligned}
$$

where the first four terms are identical to the all-electron terms in Eqs. (1) and (2) and with

$$
V_{\mu}^{\mathrm{DK} 3-\mathrm{AIMP}}=V_{\mathrm{Coul}}^{\mu, \mathrm{MP}}+V_{\mathrm{Exch}}^{\mu, \mathrm{MP}}+P_{\text {core }}^{\mu} .
$$

The $V_{\text {Coul }}$ operator represents the Coulomb interactions of one valence electron with the $Z_{\text {core }}$ electrons and the same number of protons located at the nucleus. It is convenient to fit $V_{\text {Coul }}$ with a linear combination of Gaussian functions,

$$
\begin{aligned}
V_{\text {Coul }}(i) & =-\frac{Z_{\text {core }}}{r_{i}}+2 \sum_{c}^{\text {core }} J_{c}(i) \\
& \approx V_{\text {Coul }}^{\mathrm{MP}}(i)=\sum_{j} C_{j} \frac{\mathrm{e}^{-\alpha_{j} r_{i}^{2}}}{r_{i}} .
\end{aligned}
$$

In the same way, the $V_{\text {exch }}$ operator represents the exchange interaction between one valence electron and the core electrons. Its spectral representation operator replaces this operator,

$$
\begin{aligned}
V_{\text {exch }}(i) & =-\sum_{c}^{\text {core }} K_{c}(i) \\
& \approx V_{\text {exch }}^{\mathrm{MP}}(i)=\sum_{l} \sum_{m=-l}^{l} \sum_{a, b}|a ; l m\rangle A_{l ; a b}\langle b ; l m|,
\end{aligned}
$$

where $a$ and $b$ are arbitrary Gaussian functions. $A_{l, a b}$ is an element in the following matrix:

$$
A=S^{-1} K S^{-1} \text {, where } K_{i j}=\left\langle i\left|V_{\text {exch }}\right| j\right\rangle \text { and } S_{i j}=\langle i \mid j\rangle \text {. }
$$

By using these operators, all of one-electron exchange integrals become those of the all-electron calculation, if $\langle i|$ and $|j\rangle$ belong to the $\{a ; l m\}$ and $\{b ; l m\}$ basis functions. The operator $P(i)$ is obtained by the core-valence orthogonality, and consists of the core orbitals, $\phi_{c}$, and the core orbital energies, $\epsilon_{c}$. The nodal structures of the valence orbitals are ensured through this operator:

$$
P(i)=-\sum_{c}^{\text {core }} 2 \epsilon_{c}\left|\phi_{c}\right\rangle\left\langle\phi_{c}\right| .
$$

In this way, the direct relativistic effects on the valence electrons are handled with the no-pair relativistic operators of kinetic (and rest mass) energy and nuclear attraction and the indirect relativistic effects on the valence electrons brought about by the core electrons are considered by means of the core AIMP.

\section{RESULTS}

\section{A. Atomic results}

The valence configuration $6 s^{2} 6 p^{6} 5 f^{1} 6 d^{2} 7 s^{1}$ for Th, $6 s^{2} 6 p^{6} 5 f^{n+1} 6 d^{1} 7 s^{1}$ for $\mathrm{Pa}, \mathrm{Am}$, and $6 s^{2} 6 p^{6} 5 f^{n} 6 d^{1} 7 s^{2}$ for $\mathrm{U}$ to $\mathrm{Pu}$ and $\mathrm{Cm}$ to $\mathrm{Lr}$, where $n$ stands for the position number of the respective atom in the actinide series $(\mathrm{Pa}=2$ to $\mathrm{Lr}=14$ ), were chosen as the reference states. The all-electron SCF calculations with the scalar relativistic DK3 were performed on these reference states with the relativistic uncontracted GTO. ${ }^{26}$ The orbitals and the orbital energies from these reference calculations were adopted in the present AIMP method to describe the inner core orbitals.

According to its basic approximations, the AIMP method should work when the frozen-core approximation itself works. Furthermore, the accuracy improves systematically with the quality of the valence basis set. It has been found that the AIMP perform best with the all-electron basis sets. We examined the partitioning of the core and valence orbit- 
als. Numerical experience suggests two cores, i.e., the large $[\mathrm{Xe}, 4 f, 5 d]$ and the small $[\mathrm{Xe}, 4 f]$ cores. That is, the $6 s, 6 p, 5 f, 6 d$, and $7 s$ electrons are treated as the valence electrons in the large core set while keeping 78 electrons as the core electrons. In the case of the small cores, the $5 d$ electrons are also treated as valence electrons and 68 electrons are treated as the core electrons.

The core 78 or 68 electrons were replaced with the potential. The linear combination of the 14 and 15 terms of the Gaussian-type functions were fitted to the Coulomb potentials calculated from the reference core orbitals. The exponents of the valence basis sets were optimized for the respective states by means of the minimization of the DK3 valence SCF total energies. The high quality of the inner part of the AIMP valence orbitals requires the use of basis sets with a relatively large number of primitive functions as compared with the nodeless pseudopotentials. This fact, however, does not represent a real increment in the computing time in molecular calculations. The computational cost depends on the number of contracted functions rather than on the length of their expansion. The number of basis functions (contracted functions) in AIMP molecular calculations is more or less the same as in corresponding pseudopotential calculations. The bonus of the inner quality of the valence orbitals should be expected in calculations of valence correlation energies and in spin-orbit effects.

The valence basis sets were contracted using the DK3 SCF valence orbital coefficients, and the outermost four $s$-type, four $p$-type, four $d$-type, and three $f$-type primitives were augmented to give more flexibility to the orbitals in the molecular calculations. The resulting valence basis sets for Th to $\mathrm{Lr}$ are $(14 s 10 p 11 d 9 f) /[6 s 5 p 5 d 4 f]$ for a large core $[\mathrm{Xe}, 4 f, 5 d]$ and $(14 s 10 p 12 d 9 f) /[6 s 5 p 6 d 4 f]$ for a small core, i.e., $[\mathrm{Xe}, 4 f]$.

The atomic valence properties, the orbital energies $(\epsilon)$, and radial expectation values $(\langle r\rangle)$ are calculated with the present AIMP and the valence basis set in a fully uncontracted manner. The results with a large core are given in Table I and those with a small core are shown in Table II. The present DK3-AIMP results with small and large cores are very similar and both results reproduce the all-electron calculation to a high accuracy. The mean absolute errors (MAE) for the small and large core sets are 0.003 and 0.001 hartree for the orbital energies and 0.004 and 0.006 bohr for the radial expectation values. Note that the fitting of the valence orbitals is not included in the optimization procedure for either the core potentials or the valence basis sets. Nevertheless, the present AIMP satisfactorily reproduces the valence orbitals to an excellent accuracy in the atomic calculations. The $r^{2} R(r)^{2}$ functions for the valence orbitals of the Th atom are plotted in Fig. 1. The difference between the AIMP and all-electron results is too small to be visible for the entire $r$ range on the scale of the figure.

Both small and large cores give almost identical values for all actinide atoms. We assume that the $5 d$ electrons are not valence electrons, and thus can be safely frozen into the core. That is, the $6 s, 6 p, 5 f, 6 d$ and $7 s$ electrons are expected to play an active role in actinide chemistry.

\section{B. Molecular calculations on ThO}

The spectroscopic properties, namely the dissociation energy $D_{e}$, the rotational constant $B_{e}$, the vibrational wave number $\omega_{e}$, and the bond length $r_{e}$, were calculated for the ${ }^{1} \Sigma^{+}$ground state of thorium monoxide, ThO, using the SCF and CASSCF methods. To obtain the bond length $r_{e}$, the total energy was calculated for several Th-O distances, differing by 0.05 a.u. Then the minimum has been obtained by fitting a third-degree polynomial to the eight points of lowest energy. The same potential has been used to calculate $\omega_{e}$ and $B_{e}$. The dissociation energy $D_{e}$ was subtracted from the corresponding atomic ground states of the Th $6 d^{2} 7 s^{2}{ }^{3} \mathrm{~F}, \mathrm{O} 2 p^{4}$ ${ }^{3} \mathrm{P}$, and molecular calculations of the ${ }^{1} \Sigma^{+}$ground state of $\mathrm{ThO}, \mathrm{Th}^{2+} 7 s^{2} \mathrm{O}^{2-} 2 p^{6}$, respectively.

Our calculations were carried out in $\mathrm{C}_{2}$ symmetry, using the program system MOLCAS5 ${ }^{27}$ modified for the inclusion of the DK3-AIMP. The active space in CASSCF was chosen to include eight electrons distributed over nine orbitals ( $7 s$ and $6 d$ of thorium, $2 p$ of oxygen) while the $2 s$ orbital of oxygen was kept doubly occupied.

We used two different cores for the thorium atom, the large core $[\mathrm{Xe}, 4 f, 5 d]$ with the $(14 s 10 p 11 d 9 f) /$ $[6 s 5 p 5 d 4 f]$ valence basis set and the small core [Xe, $4 f]$ with the $(14 s 10 p 12 d 9 f) /[6 s 5 p 6 d 4 f]$ valence basis set. For the oxygen atom we have produced the same kind of DK3AIMP, i.e., a [He] core with the $(6 s 7 p 1 d) /[3 s 4 p 1 d]$ valence basis set. The linear combination of the 12 terms of the Gaussian-type functions was fitted to the Coulomb potential, and the $(6 s 6 p)$ valence basis set was optimized in the oxygen ${ }^{3} \mathrm{P}$ ground state. The valence basis set was contracted into the atomic DK3 SCF valence orbitals, and the outermost two $s$-type and three $p$-type primitives were added. This basis set was further augmented by one $p$-type Gaussian primitive with the exponent $0.059,{ }^{28}$ and one $d$-type Gaussian primitive with the exponent $1.154,{ }^{29}$ representing the diffuse and the polarization functions, resulting in a $(6 s 7 p 1 d) /[3 s 4 p 1 d]$ valence basis set. The basis set employed in all-electron DK3 calculations were the relativistic GTO basis sets taken from Ref. 26, and augmented in the same way in the valence region: $(12 s 9 p 1 d) /[4 s 4 p 1 d]$ for oxygen and $(35 s 26 p 18 d 13 f) /[11 s 9 p 8 d 5 f]$ for thorium.

The calculated results are listed in Table III. The previous calculations and experimental data are also listed for comparison. Careful examination of the table shows that the AIMP satisfactorily reproduces the all-electron results and there is no significant difference in the quality between the AIMP with a small core and that with a large core.

The AIMP-SCF with a large core, $[\mathrm{Xe}, 4 f, 5 d]$ gives a bond length of $1.833 \AA$ and the AIMP-CASSCF yields $r_{e}$ $=1.879 \AA$. The SCF calculation yields a bond length slightly shorter, while the CASSCF calculation gives a longer bond length relative to experiment. However, AIMP reproduces the all-electron bond length at both levels of theory. The difference is only 0.001 and $0.002 \AA$ at the SCF and CASSCF levels, respectively. The AIMP gives vibrational frequencies that are close to the all-electron values. The difference between AIMP and all-electron calculations is 14 (SCF) and $10 \mathrm{~cm}^{-1}$ (CASSCF). This is also true for the rotational constant. The difference between AIMP and all- 
TABLE I. The spin-free relativistic valence orbital energies $(\epsilon)$ and radial expectation values $(\langle r\rangle)$ both in the DK3-AIMP and all-electron (AE) calculations. The DK3-AIMP calculations correspond to a large $[\mathrm{Xe}, 4 f, 5 d]$ core and a $6 s, 6 p, 5 f, 6 d$, and $7 s$ valence.

\begin{tabular}{|c|c|c|c|c|c|c|c|}
\hline & & & & & & & \\
\hline & Configu & & & $\begin{array}{c}\epsilon \\
\text { (Hartree) }\end{array}$ & $\begin{array}{c}\langle r\rangle \\
\text { (Bohr) }\end{array}$ & $\begin{array}{c}\epsilon \\
\text { (Hartree) }\end{array}$ & $\begin{array}{c}\langle r\rangle \\
\text { (Bohr) }\end{array}$ \\
\hline Th & $5 f^{1} 6 d^{2} 7 s^{1}$ & ${ }^{5} \mathrm{I}$ & $6 s$ & -1.907 & 1.589 & -1.912 & 1.585 \\
\hline & & & $6 p$ & -0.964 & 1.931 & -0.968 & 1.923 \\
\hline & & & $5 f$ & -0.180 & 1.723 & -0.180 & 1.713 \\
\hline & & & $6 d$ & -0.168 & 3.501 & -0.167 & 3.502 \\
\hline & & & $7 s$ & -0.187 & 4.643 & -0.187 & 4.635 \\
\hline $\mathrm{Pa}$ & $5 f^{3} 6 d^{1} 7 s^{1}$ & ${ }^{6} \mathrm{~L}$ & $6 s$ & -1.897 & 1.563 & -1.902 & 1.558 \\
\hline & & & $6 p$ & -0.925 & 1.914 & -0.928 & 1.906 \\
\hline & & & $5 f$ & -0.176 & 1.672 & -0.174 & 1.666 \\
\hline & & & $6 d$ & -0.120 & 4.060 & -0.118 & 4.073 \\
\hline & & & $7 s$ & -0.178 & 4.783 & -0.178 & 4.755 \\
\hline $\mathrm{U}$ & $5 f^{3} 6 d^{1} 7 s^{2}$ & ${ }^{5} \mathrm{~L}$ & $6 s$ & -2.123 & 1.501 & -2.126 & 1.499 \\
\hline & & & $6 p$ & -1.069 & 1.829 & -1.072 & 1.824 \\
\hline & & & $5 f$ & -0.397 & 1.438 & -0.390 & 1.441 \\
\hline & & & $6 d$ & -0.229 & 2.978 & -0.228 & 2.969 \\
\hline & & & $7 s$ & -0.196 & 4.389 & -0.196 & 4.391 \\
\hline $\mathrm{Np}$ & $5 f^{4} 6 d^{1} 7 s^{2}$ & ${ }^{6} \mathrm{~L}$ & $6 s$ & -2.203 & 1.466 & -2.206 & 1.464 \\
\hline & & & $6 p$ & -1.098 & 1.791 & -1.102 & 1.786 \\
\hline & & & $5 f$ & -0.451 & 1.356 & -0.443 & 1.359 \\
\hline & & & $6 d$ & -0.234 & 2.910 & -0.233 & 2.902 \\
\hline & & & $7 s$ & -0.199 & 4.335 & -0.199 & 4.331 \\
\hline $\mathrm{Pu}$ & $5 f^{5} 6 d^{1} 7 s^{2}$ & ${ }^{7} \mathrm{~K}$ & $6 s$ & -2.297 & 1.431 & -2.298 & 1.431 \\
\hline & & & $6 p$ & -1.138 & 1.752 & -1.142 & 1.749 \\
\hline & & & $5 f$ & -0.501 & 1.278 & -0.491 & 1.283 \\
\hline & & & $6 d$ & -0.184 & 3.170 & -0.183 & 3.170 \\
\hline & & & $7 s$ & -0.208 & 4.230 & -0.208 & 4.227 \\
\hline $\mathrm{Am}$ & $5 f^{7} 6 d^{1} 7 s^{1}$ & ${ }^{10} \mathrm{D}$ & $6 s$ & -2.205 & 1.422 & -2.210 & 1.420 \\
\hline & & & $6 p$ & -1.034 & 1.761 & -1.039 & 1.755 \\
\hline & & & $5 f$ & -0.374 & 1.288 & -0.368 & 1.291 \\
\hline & & & $6 d$ & -0.106 & 4.252 & -0.105 & 4.241 \\
\hline & & & $7 s$ & -0.194 & 4.467 & -0.195 & 4.475 \\
\hline $\mathrm{Cm}$ & $5 f^{7} 6 d^{1} 7 s^{2}$ & ${ }^{9} \mathrm{D}$ & $6 s$ & -2.441 & 1.374 & -2.447 & 1.371 \\
\hline & & & $6 p$ & -1.183 & 1.692 & -1.188 & 1.687 \\
\hline & & & $5 f$ & -0.600 & 1.186 & -0.592 & 1.188 \\
\hline & & & $6 d$ & -0.212 & 2.901 & -0.210 & 2.894 \\
\hline & & & $7 s$ & -0.210 & 4.147 & -0.210 & 4.138 \\
\hline $\mathrm{Bk}$ & $5 f^{8} 6 d^{1} 7 s^{2}$ & ${ }^{8} \mathrm{H}$ & $6 s$ & -2.543 & 1.344 & -2.541 & 1.341 \\
\hline & & & $6 p$ & -1.218 & 1.661 & -1.223 & 1.655 \\
\hline & & & $5 f$ & -0.610 & 1.151 & -0.602 & 1.153 \\
\hline & & & $6 d$ & -0.191 & 2.990 & -0.190 & 2.983 \\
\hline & & & $7 s$ & -0.216 & 4.065 & -0.216 & 4.060 \\
\hline $\mathrm{Cf}$ & $5 f^{9} 6 d^{1} 7 s^{2}$ & ${ }^{7} \mathrm{~K}$ & $6 s$ & -2.624 & 1.317 & -2.633 & 1.314 \\
\hline & & & $6 p$ & -1.252 & 1.630 & -1.257 & 1.626 \\
\hline & & & $5 f$ & -0.639 & 1.113 & -0.627 & 1.116 \\
\hline & & & $6 d$ & -0.173 & 3.114 & -0.172 & 3.115 \\
\hline & & & $7 s$ & -0.222 & 3.986 & -0.223 & 3.979 \\
\hline Es & $5 f^{10} 6 d^{1} 7 s^{2}$ & ${ }^{6} \mathrm{~L}$ & $6 s$ & -2.712 & 1.290 & -2.720 & 1.287 \\
\hline & & & $6 p$ & -1.279 & 1.603 & -1.285 & 1.599 \\
\hline & & & $5 f$ & -0.666 & 1.082 & -0.655 & 1.085 \\
\hline & & & $6 d$ & -0.169 & 3.106 & -0.168 & 3.118 \\
\hline & & & $7 s$ & -0.226 & 3.925 & -0.227 & 3.920 \\
\hline $\mathrm{Fm}$ & $5 f^{11} 6 d^{1} 7 s^{2}$ & & $6 s$ & -2.799 & 1.265 & -2.808 & 1.262 \\
\hline & & & $6 p$ & -1.305 & 1.578 & -1.312 & 1.573 \\
\hline
\end{tabular}


TABLE I. (Continued.)

\begin{tabular}{|c|c|c|c|c|c|c|c|}
\hline & & & & & & & \\
\hline & Configu & & & $\begin{array}{c}\epsilon \\
\text { (Hartree) }\end{array}$ & $\begin{array}{c}\langle r\rangle \\
\text { (Bohr) }\end{array}$ & $\begin{array}{c}\epsilon \\
\text { (Hartree) }\end{array}$ & $\begin{array}{c}\langle r\rangle \\
\text { (Bohr) }\end{array}$ \\
\hline & & & $5 f$ & -0.692 & 1.053 & -0.679 & 1.055 \\
\hline & & & $6 d$ & -0.165 & 3.115 & -0.164 & 3.113 \\
\hline & & & $7 s$ & -0.231 & 3.865 & -0.231 & 3.862 \\
\hline Md & $5 f^{12} 6 d^{1} 7 s^{2}$ & ${ }^{4} \mathrm{~K}$ & $6 s$ & -2.889 & 1.240 & -2.889 & 1.237 \\
\hline & & & $6 p$ & -1.332 & 1.553 & -1.339 & 1.548 \\
\hline & & & $5 f$ & -0.716 & 1.026 & -0.704 & 1.028 \\
\hline & & & $6 d$ & -0.161 & 3.132 & -0.159 & 3.131 \\
\hline & & & $7 s$ & -0.235 & 3.811 & -0.236 & 3.800 \\
\hline No & $5 f^{13} 6 d^{1} 7 s^{2}$ & ${ }^{3} \mathrm{H}$ & $6 s$ & -2.978 & 1.217 & -2.992 & 1.213 \\
\hline & & & $6 p$ & -1.358 & 1.530 & -1.366 & 1.525 \\
\hline & & & $5 f$ & -0.744 & 1.000 & -0.732 & 1.002 \\
\hline & & & $6 d$ & -0.156 & 3.153 & -0.155 & 3.166 \\
\hline & & & $7 s$ & -0.240 & 3.752 & -0.241 & 3.746 \\
\hline Lr & $5 f^{14} 6 d^{1} 7 s^{2}$ & ${ }^{2} \mathrm{D}$ & $6 s$ & -3.070 & 1.195 & -3.085 & 1.191 \\
\hline & & & $6 p$ & -1.382 & 1.509 & -1.391 & 1.503 \\
\hline & & & $5 f$ & -0.775 & 0.976 & -0.766 & 0.978 \\
\hline & & & $6 d$ & -0.151 & 3.189 & -0.149 & 3.203 \\
\hline & & & $7 s$ & -0.245 & 3.695 & -0.246 & 3.688 \\
\hline
\end{tabular}

TABLE II. The spin-free relativistic valence orbital energies $(\epsilon)$ and radial expectation values $(\langle r\rangle)$ both in the DK3-AIMP and all-electron (AE) calculations. The DK3-AIMP calculations correspond to a small [Xe, $4 f]$ core and a $5 d, 6 s, 6 p, 5 f, 6 d$, and $7 s$ valence.

\begin{tabular}{|c|c|c|c|c|c|c|c|}
\hline & & & & \multicolumn{2}{|c|}{ AIMP } & \multicolumn{2}{|c|}{$\mathrm{AE}$} \\
\hline \multicolumn{4}{|c|}{ Configuration } & $\begin{array}{c}\epsilon \\
\text { (Hartree) }\end{array}$ & $\begin{array}{c}\langle r\rangle \\
(\text { Bohr })\end{array}$ & $\begin{array}{c}\epsilon \\
\text { (Hartree) }\end{array}$ & $\begin{array}{c}\langle r\rangle \\
\text { (Bohr) }\end{array}$ \\
\hline \multirow[t]{6}{*}{$\mathrm{Th}$} & $5 f^{1} 6 d^{2} 7 s^{1}$ & ${ }^{5} \mathrm{I}$ & $5 d$ & -3.642 & 0.934 & -3.639 & 0.933 \\
\hline & & & $6 s$ & -1.911 & 1.588 & -1.912 & 1.585 \\
\hline & & & $6 p$ & -0.965 & 1.928 & -0.968 & 1.923 \\
\hline & & & $5 f$ & -0.185 & 1.708 & -1.180 & 1.713 \\
\hline & & & $6 d$ & -0.167 & 3.500 & -0.167 & 3.502 \\
\hline & & & $7 s$ & -0.187 & 4.644 & -0.187 & 4.635 \\
\hline \multirow[t]{6}{*}{$\mathrm{Pa}$} & $5 f^{3} 6 d^{1} 7 s^{1}$ & ${ }^{6} \mathrm{~L}$ & $5 d$ & -3.754 & 0.913 & -3.751 & 0.912 \\
\hline & & & $6 s$ & -1.900 & 1.562 & -1.902 & 1.558 \\
\hline & & & $6 p$ & -0.925 & 1.912 & -0.928 & 1.906 \\
\hline & & & $5 f$ & -0.180 & 1.662 & -0.174 & 1.666 \\
\hline & & & $6 d$ & -0.119 & 4.076 & -0.118 & 4.073 \\
\hline & & & $7 s$ & -0.178 & 4.784 & -0.178 & 4.755 \\
\hline \multirow[t]{6}{*}{$\mathrm{U}$} & $5 f^{3} 6 d^{1} 7 s^{2}$ & ${ }^{5} \mathrm{~L}$ & $5 d$ & -4.159 & 0.886 & -4.155 & 0.886 \\
\hline & & & $6 s$ & -2.123 & 1.501 & -2.126 & 1.499 \\
\hline & & & $6 p$ & -1.069 & 1.829 & -1.072 & 1.824 \\
\hline & & & $5 f$ & -0.397 & 1.438 & -0.390 & 1.441 \\
\hline & & & $6 d$ & -0.229 & 2.977 & -0.228 & 2.969 \\
\hline & & & $7 s$ & -0.196 & 4.389 & -0.196 & 4.391 \\
\hline \multirow[t]{6}{*}{$\mathrm{Np}$} & $5 f^{4} 6 d^{1} 7 s^{2}$ & ${ }^{6} \mathrm{~L}$ & $5 d$ & -4.387 & 0.865 & -4.384 & 0.865 \\
\hline & & & $6 s$ & -2.204 & 1.466 & -2.206 & 1.464 \\
\hline & & & $6 p$ & -1.098 & 1.791 & -1.102 & 1.786 \\
\hline & & & $5 f$ & -0.450 & 1.356 & -0.443 & 1.359 \\
\hline & & & $6 d$ & -0.234 & 2.906 & -0.233 & 2.902 \\
\hline & & & $7 s$ & -0.199 & 4.334 & -0.199 & 4.331 \\
\hline \multirow[t]{4}{*}{$\mathrm{Pu}$} & $5 f^{5} 6 d^{1} 7 s^{2}$ & ${ }^{7} \mathrm{~K}$ & $5 d$ & -4.623 & 0.846 & -4.621 & 0.845 \\
\hline & & & $6 s$ & -2.294 & 1.432 & -2.298 & 1.431 \\
\hline & & & $6 p$ & -1.137 & 1.753 & -1.142 & 1.749 \\
\hline & & & $5 f$ & -0.498 & 1.280 & -0.491 & 1.283 \\
\hline
\end{tabular}


TABLE II. (Continued.)

\begin{tabular}{|c|c|c|c|c|c|c|c|}
\hline \multirow{2}{*}{\multicolumn{2}{|c|}{ Configuration }} & & & \multicolumn{2}{|c|}{ AIMP } & \multicolumn{2}{|c|}{$\mathrm{AE}$} \\
\hline & & & & $\begin{array}{c}\epsilon \\
\text { (Hartree) }\end{array}$ & $\begin{array}{c}\langle r\rangle \\
\text { (Bohr) }\end{array}$ & $\begin{array}{c}\epsilon \\
\text { (Hartree) }\end{array}$ & $\begin{array}{c}\langle r\rangle \\
\text { (Bohr) }\end{array}$ \\
\hline & & & $6 d$ & -0.184 & 3.173 & -0.183 & 3.170 \\
\hline & & & $7 s$ & -0.208 & 4.228 & -0.208 & 4.227 \\
\hline \multirow[t]{6}{*}{ Am } & $5 f^{7} 6 d^{1} 7 s^{1}$ & ${ }^{10} \mathrm{D}$ & $5 d$ & -4.644 & 0.830 & -4.643 & 0.829 \\
\hline & & & $6 s$ & -2.206 & 1.422 & -2.210 & 1.420 \\
\hline & & & $6 p$ & -1.034 & 1.761 & -1.039 & 1.755 \\
\hline & & & $5 f$ & -0.374 & 1.288 & -0.368 & 1.291 \\
\hline & & & $6 d$ & -0.106 & 4.244 & -0.105 & 4.241 \\
\hline & & & $7 s$ & -0.194 & 4.481 & -0.195 & 4.475 \\
\hline \multirow[t]{6}{*}{$\mathrm{Cm}$} & $5 f^{7} 6 d^{1} 7 s^{2}$ & ${ }^{9} \mathrm{D}$ & $5 d$ & -5.064 & 0.809 & -5.061 & 0.809 \\
\hline & & & $6 s$ & -2.441 & 1.374 & -2.447 & 1.371 \\
\hline & & & $6 p$ & -1.183 & 1.692 & -1.188 & 1.687 \\
\hline & & & $5 f$ & -0.600 & 1.186 & -0.592 & 1.188 \\
\hline & & & $6 d$ & -0.211 & 2.904 & -0.210 & 2.894 \\
\hline & & & $7 s$ & -0.210 & 4.143 & -0.210 & 4.138 \\
\hline \multirow[t]{6}{*}{$\mathrm{Bk}$} & $5 f^{8} 6 d^{1} 7 s^{2}$ & ${ }^{8} \mathrm{H}$ & $5 d$ & -5.305 & 0.793 & -5.302 & 0.792 \\
\hline & & & $6 s$ & -2.535 & 1.344 & -2.541 & 1.341 \\
\hline & & & $6 p$ & -1.218 & 1.661 & -1.223 & 1.655 \\
\hline & & & $5 f$ & -0.610 & 1.150 & -0.602 & 1.153 \\
\hline & & & $6 d$ & -0.191 & 2.993 & -0.190 & 2.983 \\
\hline & & & $7 s$ & -0.216 & 4.063 & -0.216 & 4.060 \\
\hline \multirow[t]{6}{*}{$\mathrm{Cf}$} & $5 f^{9} 6 d^{1} 7 s^{2}$ & ${ }^{7} \mathrm{~K}$ & $5 d$ & -5.541 & 0.777 & -5.540 & 0.776 \\
\hline & & & $6 s$ & -2.626 & 1.316 & -2.633 & 1.314 \\
\hline & & & $6 p$ & -1.250 & 1.631 & -1.257 & 1.626 \\
\hline & & & $5 f$ & -0.635 & 1.114 & -0.627 & 1.116 \\
\hline & & & $6 d$ & -0.173 & 3.121 & -0.172 & 3.115 \\
\hline & & & $7 s$ & -0.223 & 3.973 & -0.223 & 3.979 \\
\hline \multirow[t]{6}{*}{ Es } & $5 f^{10} 6 d^{1} 7 s^{2}$ & ${ }^{6} \mathrm{~L}$ & $5 d$ & -5.773 & 0.762 & -5.772 & 0.761 \\
\hline & & & $6 s$ & -2.713 & 1.290 & -2.720 & 1.287 \\
\hline & & & $6 p$ & -1.278 & 1.604 & -1.285 & 1.599 \\
\hline & & & $5 f$ & -0.662 & 1.083 & -0.655 & 1.085 \\
\hline & & & $6 d$ & -0.169 & 3.115 & -0.168 & 3.118 \\
\hline & & & $7 s$ & -0.227 & 3.926 & -0.227 & 3.920 \\
\hline \multirow[t]{6}{*}{ Fm } & $5 f^{11} 6 d^{1} 7 s^{2}$ & ${ }^{5} \mathrm{~L}$ & $5 d$ & -6.005 & 0.747 & -6.004 & 0.747 \\
\hline & & & $6 s$ & -2.801 & 1.264 & -2.808 & 1.262 \\
\hline & & & $6 p$ & -1.305 & 1.578 & -1.312 & 1.573 \\
\hline & & & $5 f$ & -0.688 & 1.054 & -0.679 & 1.055 \\
\hline & & & $6 d$ & -0.165 & 3.132 & -0.164 & 3.113 \\
\hline & & & $7 s$ & -0.231 & 3.862 & -0.231 & 3.862 \\
\hline \multirow[t]{6}{*}{ Md } & $5 f^{12} 6 d^{1} 7 s^{2}$ & ${ }^{4} \mathrm{~K}$ & $5 d$ & -6.239 & 0.733 & -6.237 & 0.733 \\
\hline & & & $6 s$ & -2.889 & 1.240 & -2.899 & 1.237 \\
\hline & & & $6 p$ & -1.331 & 1.554 & -1.339 & 1.548 \\
\hline & & & $5 f$ & -0.713 & 1.026 & -0.704 & 1.028 \\
\hline & & & $6 d$ & -0.161 & 3.137 & -0.159 & 3.131 \\
\hline & & & $7 s$ & -0.236 & 3.809 & -0.236 & 3.800 \\
\hline \multirow[t]{6}{*}{ No } & $5 f^{13} 6 d^{1} 7 s^{2}$ & ${ }^{3} \mathrm{H}$ & $5 d$ & -6.472 & 0.720 & -6.471 & 0.720 \\
\hline & & & $6 s$ & -2.980 & 1.217 & -2.992 & 1.213 \\
\hline & & & $6 p$ & -1.357 & 1.531 & -1.366 & 1.525 \\
\hline & & & $5 f$ & -0.741 & 1.001 & -0.732 & 1.002 \\
\hline & & & $6 d$ & -0.156 & 3.158 & -0.155 & 3.166 \\
\hline & & & $7 s$ & -0.240 & 3.757 & -0.241 & 3.746 \\
\hline \multirow[t]{6}{*}{$\mathrm{Lr}$} & $5 f^{14} 6 d^{1} 7 s^{2}$ & ${ }^{2} \mathrm{D}$ & $5 d$ & -6.705 & 0.707 & -6.703 & 0.707 \\
\hline & & & $6 s$ & -3.073 & 1.194 & -3.085 & 1.191 \\
\hline & & & $6 p$ & -1.382 & 1.509 & -1.391 & 1.503 \\
\hline & & & $5 f$ & -0.776 & 0.976 & -0.766 & 0.978 \\
\hline & & & $6 d$ & -0.150 & 3.194 & -0.149 & 3.203 \\
\hline & & & $7 s$ & -0.245 & 3.694 & -0.246 & 3.688 \\
\hline
\end{tabular}




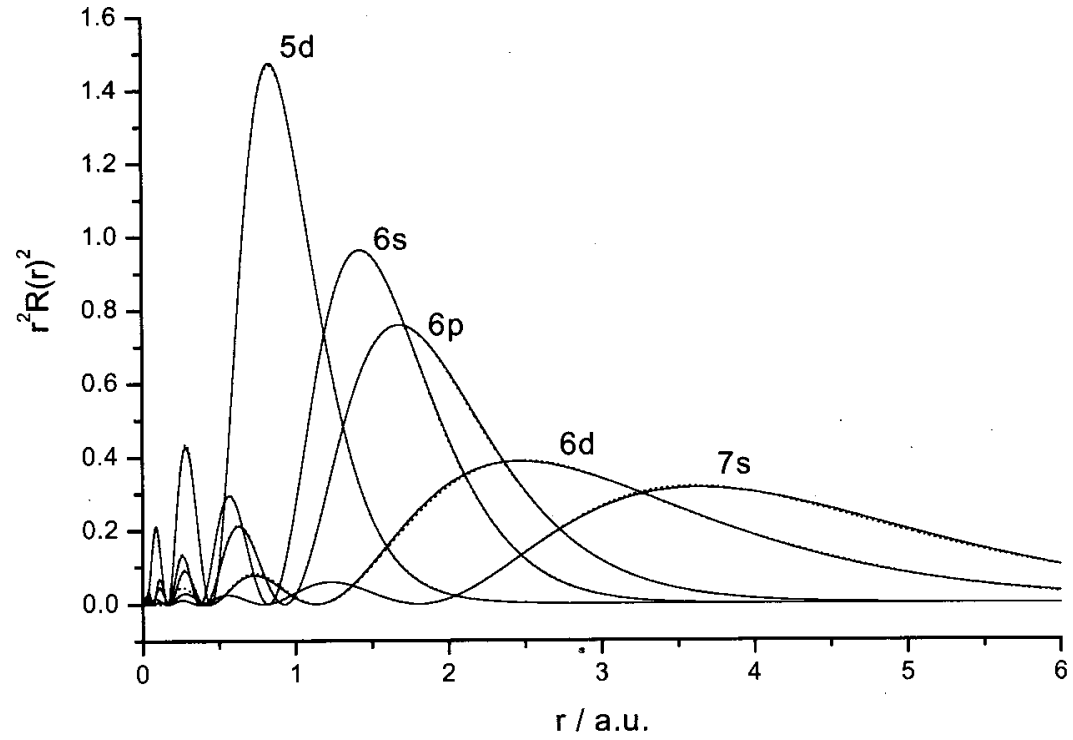

FIG. 1. The $r^{2} R(r)^{2}$ functions for the $5 d, 6 s, 6 p, 5 f, 6 d, 7 s$ orbitals for the corresponding small and large core sets of the excited state of $\left({ }^{5} \mathrm{I}\right) \mathrm{Th}$. The solid and dotted lines denote the AIMP and the all-electron (AE) results, respectively. electron calculations is only $0.001 \mathrm{~cm}^{-1}$. The observed dissociation energy is $9.00 \mathrm{eV}$ for ThO. The $\mathrm{SCF} D_{e}$ is very poor compared with the experimental value. However, the AIMP-SCF $D_{e}$ of $5.96 \mathrm{eV}$ is close to the all-electron SCF $D_{e}$ of $5.82 \mathrm{eV}$. The CASSCF improves the deficiency of the SCF and gives a value close to the observed one. Again, the AIMP-CASSCF value of $9.14 \mathrm{eV}$ is close to the all-electron value of $9.01 \mathrm{eV}$. The present calculations show that the

TABLE III. Spectroscopic constants for the ${ }^{1} \Sigma^{+}$ground state of thorium monoxide, ThO, using the DK3-AIMP and corresponding all-electron DK3 (AE-DK3) methods.

\begin{tabular}{lcccc}
\hline \hline & $\begin{array}{c}r_{e} \\
(\AA)\end{array}$ & $\begin{array}{c}\omega_{e} \\
\left(\mathrm{~cm}^{-1}\right)\end{array}$ & $\begin{array}{c}B_{e} \\
\left(\mathrm{~cm}^{-1}\right)\end{array}$ & $\begin{array}{c}D_{e} \\
(\mathrm{eV})\end{array}$ \\
\hline $\begin{array}{l}\text { This work } \\
\text { AIMP-SCF }\end{array}$ & 1.833 & 945 & 0.335 & 5.96 \\
AIMP-CASSCF $^{\mathrm{a}}$ & 1.879 & 856 & 0.319 & 9.14 \\
AIMP-SCF $^{\mathrm{b}}$ & 1.832 & 951 & 0.336 & 5.96 \\
AIMP-CASSCF $^{\mathrm{b}}$ & 1.878 & 861 & 0.320 & 9.14 \\
& & & & \\
AE-SCF $^{\mathrm{c}}$ & 1.832 & 959 & 0.336 & 5.82 \\
AE-CASSCF $^{\mathrm{c}}$ & 1.877 & 866 & 0.320 & 9.01 \\
Previously $^{\text {AIMP-SCF }}$ & & & & \\
AIMP-CASSCF $^{\mathrm{d}}$ & 1.819 & 956 & & 5.99 \\
PP-SCF $^{\mathrm{e}}$ & 1.886 & 865 & & 9.15 \\
PP-CASSCF $^{\mathrm{e}}$ & 1.829 & 943 & & 6.07 \\
AE-DFR $^{\mathrm{f}}$ & 1.882 & 876 & & 8.92 \\
Expt. $^{\mathrm{g}}$ & 1.873 & 930 & 0.321 & 7.67 \\
\hline
\end{tabular}

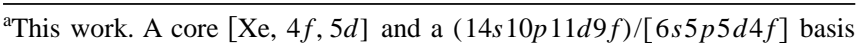
set.

${ }^{\mathrm{b}}$ This work. A core $[\mathrm{Xe}, 4 f]$ and a $(14 s 10 p 12 d 9 f) /[6 s 5 p 6 d 4 f]$ basis set. ${ }^{\mathrm{c}}$ This work. A $(35 s 26 p 18 d 13 f) /[11 s 9 p 8 d 5 f]$ basis set.

${ }^{\mathrm{d}}$ Reference 30. A [Xe, $\left.4 f, 5 d\right]$ core and a $(14 s 10 p 11 d 9 f) /[6 s 5 p 5 d 5 f]$ Cowan-Griffin basis set.

${ }^{\mathrm{e}}$ Reference 12. Relativistic energy-adjusted pseudopotential calculation corresponding to a $[\mathrm{Kr}, 4 d, 4 f]$ core and a $(12 s 11 p 10 d 8 f) /[8 s 7 p 6 d 4 f]$ basis set.

${ }^{\mathrm{f}}$ Reference 31. All-electron Dirac-Fock-Roothaan calculations with a $(24 s 19 p 16 d 8 f) /[8 s 7 p 7 d 5 f]$ basis set.

${ }^{\mathrm{g}}$ Reference 32.
AIMP with a large core, $[\mathrm{Xe}, 4 f, 5 d]$ works well at the SCF and CASSCF levels.

As expected, spectroscopic properties calculated by AIMP with a small core $[\mathrm{Xe}, 4 f]$ are very close to the allelectron values. The AIMP values agree with the all-electron ones within $0.001 \AA$ in $r_{e}, 8 \mathrm{~cm}^{-1}$ in $\omega_{e}$, and $0.001 \mathrm{~cm}^{-1}$ in $B_{e}$. The dissociation energy reproduces the all-electron value with an error of less than $0.14 \mathrm{eV}$.

The present calculations confirm that the DK3-AIMP works quite well, and that the errors relative to the allelectron calculations are acceptable. In addition, the large core gives a similar accuracy to the small core, leading to the conclusion that the $5 d$ electrons can be treated as core electrons in actinide chemistry.

The table also lists the previous results calculated with the Cowan-Griffin AIMP $^{30}$ and the relativistic energyadjusted pseudopotential. ${ }^{12}$ The Cowan-Griffin AIMP employs the same core-valence partitioning as the present large core while the energy-adjusted pseudopotential uses a smaller core than the present treatment. It is difficult to discuss the quality of these ECPs from the limited data. However, all calculations show very similar tendencies for the spectroscopic properties of ThO. The table also includes the all-electron Dirac-Hartree-Fock results. ${ }^{31}$ Dirac-HartreeFock includes the spin-dependent effects such as the spinorbit effect, which is not considered in the present treatment. A comparison with our AIMP-SCF results suggests that the spin-orbit effect is significant, particularly on the bond length and the dissociation energy of ThO.

\section{CONCLUSION}

We have developed the AIMP for the actinide series from Th to Lr. Two different cores, large $[\mathrm{Xe}, 4 f, 5 d]$ and small $[\mathrm{Xe}, 4 f]$ cores have been employed and the corresponding valence basis sets, $(14 s 10 p 11 d 9 f) /[6 s 5 p 5 d 4 f]$ and $(14 s 10 p 12 d 9 f) /[6 s 5 p 6 d 4 f]$, were optimized by minimizing the valence SCF total energies. The relativistic effects were considered through the scalar relativistic DK3 approxi- 
mation. The present AIMP, and the valence basis sets, were tested for the atoms and ThO. The atomic and molecular results are in excellent agreement with all-electron calculations. These illustrative calculations confirm that the present AIMP is of sufficient accuracy throughout the actinide series. The large core set gives almost the same results as the small set for atomic and molecular calculations, suggesting that the $5 d$ electrons could safely be omitted from the valence electrons in actinide chemistry.

The present AIMP can easily be combined with highly accurate relativistic effects and correlation treatments, both of which are vital for the actinides. While in this study we neglected the effect of spin-orbit coupling, which is important for $5 f$ elements, the spin-orbit effects may be included by means of mean-field and AIMP-based spin-orbit methods. ${ }^{33}$ Thus, the entire field of actinide chemistry is now open for an accurate theoretical treatment.

The present DK3-AIMP core and valence basis sets are available on AIP Document E-PAPS files. ${ }^{34}$

\section{ACKNOWLEDGMENTS}

This research was supported in part by a grant-in-aid for Scientific Research in Priority Areas "Molecular Physical Chemistry" from the Ministry of Education, Science, Culture, and Sports of Japan, and by a grant from the Genesis Research Institute. J. P. is grateful for a scholarship from the Ministry of Education, Science, Culture, and Sports of Japan under the Japanese Government Scholarship Program for Foreign Students.

${ }^{1}$ J. C. Phillips and L. Kleinman, Phys. Rev. 116, 287 (1959).

${ }^{2}$ S. Huzinaga and A. A. Cantu, J. Chem. Phys. 55, 5543 (1971).

${ }^{3}$ S. Huzinaga, D. McWilliams, and A. A. Cantu, Adv. Quantum Chem. 7, 187 (1973)

${ }^{4}$ V. Bonifacic and S. Huzinaga, J. Chem. Phys. 60, 2779 (1974).

${ }^{5}$ S. Katsuki and S. Huzinaga, Chem. Phys. Lett. 152, 203 (1988).

${ }^{6}$ Y. Sakai, E. Miyoshi, M. Klobukowski, and S. Huzinaga, J. Comput. Chem. 8, 226 (1987); 8, 256 (1987).

${ }^{7}$ S. Huzinaga, L. Seijo, Z. Barandiarán, and M. Klobukowski, J. Chem. Phys. 86, 2132 (1987).
${ }^{8}$ L. Seijo, Z. Barandiarán, and S. Huzinaga, J. Chem. Phys. 91, 7011 (1989).

${ }^{9}$ F. Rakowitz, C. M. Marian, L. Seijo, and U. Wahlgren, J. Chem. Phys. 110, 3678 (1999).

${ }^{10}$ F. Rakowitz, C. M. Marian, and L. Seijo, J. Chem. Phys. 111, 10436 (1999).

${ }^{11}$ M. Dolg, H. Stoll, and H. Preuss, J. Chem. Phys. 90, 1730 (1989).

${ }^{12}$ W. Küchle, M. Dolg, H. Stoll, and H. Preuss, J. Chem. Phys. 100, 7535 (1994).

${ }^{13}$ R. B. Ross, S. Gayen, and W. C. Ermler, J. Chem. Phys. 100, 8145 (1994).

${ }^{14}$ W. C. Ermler, R. B. Ross, and P. A. Christiansen, Int. J. Quantum Chem. 40, 829 (1991).

${ }^{15}$ T. R. Cundari and W. J. Stevens, J. Chem. Phys. 98, 5555 (1993).

${ }^{16}$ K. Motegi, T. Nakajima, K. Hirao, and L. Seijo, J. Chem. Phys. 114, 6000 (2001).

${ }^{17}$ T. Tsuchiya, T. Nakajima, K. Hirao, and L. Seijo Chem. Phys. Lett. (in press).

${ }^{18}$ P. J. Hay and W. R. Wadt, J. Chem. Phys. 82, 284 (1985).

${ }^{19}$ P. J. Hay and W. R. Wadt, J. Chem. Phys. 82, 270 (1985).

${ }^{20}$ P. J. Hay and W. R. Wadt, J. Chem. Phys. 82, 299 (1985).

${ }^{21}$ T. Nakajima and K. Hirao, J. Chem. Phys. 113, 7786 (2000).

${ }^{22}$ T. Nakajima and K. Hirao, Chem. Phys. Lett. 329, 511 (2000).

${ }^{23}$ M. Douglas and N. M. Kroll, Ann. Phys. (N.Y.) 82, 89 (1974).

${ }^{24}$ B. Hess, Phys. Rev. A 33, 3742 (1986).

${ }^{25}$ G. Jansen and B. Hess, Phys. Rev. A 39, 6016 (1989).

${ }^{26}$ T. Tsuchiya, M. Abe, T. Nakajima, and K. Hirao, J. Chem. Phys. 115, 4463 (2001).

${ }^{27}$ K. Andersson, M. Barysz, A. Bernhardsson et al., Molcas Version 5.1. Dept. of Theor. Chem., Chem. Center, Univ. of Lund, P.O. box 124, S-221 00 Lund, Sweden, Lund, 2000.

${ }^{28}$ T. H. Dunning and P. J. Hay, Modern Theoretical Chemistry, edited by H. F. Schaefer III (Plenum, New York, 1977), p. 1.

${ }^{29}$ J. Andzelm, M. Klobukowski, E. Radzio-Andzelm, Y. Sakai, and H. Tatewaki, Gaussian Basis Sets for Molecular Calculations, edited by S. Huzinaga (Elsevier, Amsterdam, 1984).

${ }^{30}$ L. Seijo, Z. Barandiarán, and E. Harguindey, J. Chem. Phys. 114, 118 (2001).

${ }^{31}$ Y. Watanab and O. Matsuoka, J. Chem. Phys. 107, 3738 (1997).

${ }^{32}$ K. P. Huber and G. Herzberg, Molecular Spectra and Molecular Structure of Diatomic Molecules (Van Nostrand, New York, 1979).

${ }^{33}$ Ch. M. Marian and U. Wahlgren, Chem. Phys. Lett. 251, 357 (1996).

${ }^{34}$ See E-PAPS Document No. E-JCPSA6-116-302227 for third-order Douglas-Kroll $a b$ initio model potential and valence basis sets for actinide elements from Th to Lr. This document may be retrieved via the EPAPS homepage (http://www.aip.org/pubservs/epaps.html) or from ftp.aip.org in the directory /epaps/. See the EPAPS homepage for more information. 